\title{
TURBULENCE WITHOUT PRESSURE
}

\author{
A.M. Polyakov \\ Physics Department, Princeton University, \\ Jadwin Hall, Princeton, NJ 08544-1000. \\ E-mail: polyakov@puhep1.princeton.edu
}

\begin{abstract}
We develop exact field theoretic methods to treat turbulence when the effect of pressure is negligible. We find explicit forms of certain probability distributions, demonstrate that the breakdown of Galilean invariance is responsible for intermittency and establish the operator product expansion. We also indicate how the effects of pressure can be turned on perturbatively.
\end{abstract}


Turbulence is an old and tantalizing subject. Enormous amounts of data and ideas have been accumulated during this century and still the problem is not solved. In our opinion, the reason lies in the fact that the necessary field-theoretic tools have appeared only recently.

Two years ago an attempt was made to apply the methods of conformal field theory to the case of two-dimensional turbulence [1]. The main concepts of this work were the following. First, one looks at the steady state condition, which relates the equal time, $\mathrm{N}$ - point and $\mathrm{N}+1$ - point functions. Then one argues that in the inertial range these relations can be solved exactly by field theories satisfying fusion rules or operator product expansions (OPE). There appeared to be infinitely many solutions. An additional constraint on these solutions follows from the constant flux conditions.

It has been noticed (although not really exploited) in [1] that there exists a striking analogy between the constant flux states in turbulence and axial (and other) anomalies in quantum field theory. The latter are violations of the naive conservation laws caused by the ultra-violet regularization. In the case of turbulence the ultraviolet regularization arises from viscosity and results in an energy flux through the inertial range. When the steady state condition with these two tools was analysed it appeared that the third ingredient was needed. Namely the physical correlation functions contained so-called condensate terms, which were $\delta$ - functions in the momentum space and represented the large scale motions of the fluid. Their role was to cancel infrared divergencies yhat arose from the field-theoretic fluctuations. Precise form of these terms depends on the large scale region were the energy is pumped into the system. The task of joining the inertial range with this region remained unsolved in [1].

It is highly desirable to have an exactly soluble model in which the above ideas can be tested at work. In this article we will discuss such a model, which also is of independent physical interest. The model in question is simply the Navier-Stokes equation with white noise random force and with the pressure set equal to zero. In one dimension this is known as the Burgers equations.

Such equations have been exploited in the past in many different physical situations (like galaxy formations [ 2 ], crystal growth [ 3 ] etc). Recently they were the subject of deep mathematical investigations [ 4 ]

In a remarkable paper [5] some striking numerical data concerning Burgers turbulence were obtained and an appealing qualitative picture of the phenomenon has been proposed. This work to a large extent inspired my interest in turbulence without pressure. Another important work in this area is the recent paper [6] on which I shall comment later.

In the present paper we shall formulate a general new method for analyzing the inertial range correlation functions, based on the ingredients mentioned above (OPE and anomalies). The method with minor modifications is also applicable to the problem of advection of passive scalars and other cases. It is obvious that the ideas we develop below will become a part of the general theory of turbulence. They may also have a considerable 
"back reaction" on the field theory.

Let us start with the one dimensional case. Burgers equation has the form:

$$
\begin{array}{r}
u_{t}+u u_{x}=\nu u_{x x}+f(x t) \\
<f(x, t) f(\dot{x}, \hat{t})=\kappa(x-\dot{x}) \delta(t-\hat{t})
\end{array}
$$

Here the function $\kappa$ defines the spatial correlation of the random forces. Consider the following generating functional:

$$
Z\left(\lambda_{1} x_{1}|\ldots| \lambda_{N} x_{N}\right)=\left\langle\exp \sum \lambda_{j} u\left(x_{j} t\right)\right\rangle
$$

From (1) we derive :

$$
\dot{Z}+\sum \lambda_{j} \frac{\partial}{\partial \lambda_{j}}\left(\frac{1}{\lambda_{j}} \frac{\partial Z}{\partial x_{j}}\right)=\sum \lambda_{j}\left\langle\left(f\left(x_{j} t\right)+\nu u^{\prime \prime}\right) \exp \sum \lambda_{k} u\left(x_{k} t\right)\right\rangle
$$

The first term in the right hand side is easy to treat since the force $f(x t)$ is Gaussian and we can use the standard trick [7] of the theory of Langevin equations:

$$
\left\langle f(x t) \exp \sum \lambda_{j} u\left(x_{j} t\right)\right\rangle=\sum \kappa\left(x-x_{j}\right) \lambda_{j} Z
$$

Our equation takes the form:

$$
\dot{Z}+\sum \lambda_{j} \frac{\partial}{\partial \lambda_{j}}\left(\frac{1}{\lambda_{j}} \frac{\partial Z}{\partial x_{j}}\right)=\sum \kappa\left(x_{i}-x_{j}\right) \lambda_{i} \lambda_{j} Z+D
$$

By $\mathrm{D}$ we denote the dissipation term:

$$
D=\nu \sum \lambda_{j}\left\langle u^{\prime \prime}\left(x_{j} t\right) \exp \sum \lambda_{k} u\left(x_{k} t\right)\right\rangle
$$

If the viscosity $\nu$ were zero our task would be completed since we have a closed differential equation for $\mathrm{Z}$. To reach the inertial range we must, however, keep $\nu$ infinitesimal but non-zero. The anomaly mechanism mentioned above implies that infinitesimal viscosity produces a finite effect, whose computation is one of our main objectives. First, however, let us transform and interpret the inviscid equations (5) (dropping the D-term).

Let us introduce the function $F$ given by :

$$
Z=\lambda_{1} \ldots \lambda_{N} F\left(\lambda_{1} x_{1} \ldots \lambda_{N} x_{N}\right)
$$

We have:

$$
\dot{F}+\sum \frac{\partial^{2}}{\partial x_{k} \partial \lambda_{k}} F-\sum \kappa\left(x_{i}-x_{j}\right) \lambda_{i} \lambda_{j} F=\tilde{D}
$$


Here:

$$
\tilde{D}=\frac{D}{\prod \lambda_{j}}
$$

We can now introduce the Fourier- transform $\mathcal{F}=\mathcal{F}\left(u_{1} x_{1} \ldots u_{N} x_{N}\right)$

which satisfies:

$$
\dot{\mathcal{F}}+\sum u_{k} \frac{\partial}{\partial x_{k}} \mathcal{F}-\sum \kappa\left(x_{i}-x_{j}\right) \frac{\partial^{2}}{\partial u_{i} \partial u_{j}} \mathcal{F}=\tilde{\mathcal{D}}
$$

obtained from(8) by the substitution $\lambda \Rightarrow \frac{\partial}{\partial u}$

The function $\mathcal{F}$ has a simple meaning. It can be interpreted as:

$$
\mathcal{F}=\left\langle\theta\left(u_{1} x_{1}\right) \ldots \theta\left(u_{N} x_{N}\right)\right\rangle
$$

where $\theta\left(u_{k} x_{k}\right)=\theta\left(u_{k}-u\left(x_{k} t\right)\right)$

and the last $\theta$ being a step function. In order to get the N-point probability distribution $Z$ one has to differentiate $\mathcal{F}$ according to $(7)$ :

$$
Z_{N}=\frac{\partial^{N}}{\partial u_{1} \ldots \partial u_{N}} \mathcal{F}_{N}
$$

We supplied here our correlation functions with the subscript $N$ to indicate the number of points on which these functions depend.

Of course, equation (9) could have been obtained directly by computing time derivative of the $\theta$ - field. It is also easy to write to express the $D_{N}$ - term through $\mathcal{F}_{N+1}$ By using (6) we obtain:

$$
\begin{gathered}
D_{N}=\sum \frac{\partial \Gamma_{j}^{(N)}}{\partial u_{j}} \\
\Gamma_{j}^{(N)}=\left.\nu \frac{\partial^{2}}{\partial y^{2}} \int u_{0} d u_{0} \frac{\partial}{\partial u_{0}} \mathcal{F}_{N+1}\left(u_{0}, x_{j}+y ; u_{1}, x_{1} \ldots u_{N}, x_{N}\right)\right|_{y \rightarrow 0}
\end{gathered}
$$

Equations (9) and (12) give a chain of relations remarkably similar to the BBGKY equations of statistical mechanics [7]. One can hardly hope to solve these equations exactly. But we are interested in the inertial range, which means that we have to take the limit $\nu \rightarrow 0$. We will show now that in this case the system of equations closes and gives us an equation for turbulent kinetics, much in a same way as the Boltzman equation becomes exact in the limit of small densities.

The main ideas of the derivation are the following. For the large Reynolds numbers, corresponding to small $\nu$ there are two relevant scales. The first, $L$, is defined by the 
size of the system and provides an infrared cut-off. The second , $a \ll L$, is the scale at which dissipation becomes relevant. The ratio $\frac{L}{a}$ goes to infinity together with the Reynolds number. By the existence of the inertial range we mean the conjecture that the correlation functions $\mathcal{F}$ have a finite limit at zero viscosity, provided that we keep $x_{i}-x_{j}$ fixed. They can have singularities at coinciding points, which must be understood as being smeared by the viscosity at the scale $a$. In fact, this scale is determined by the condition that as we let $\nu$ go to zero, dissipation remains finite. This means, that we have to find the leading singularity in (12) as $y \rightarrow 0$, and compensate for it by an appropriate scaling of $\nu(y)$. All subleading terms will give vanishing contribution in the inertial range (in the limit of the infinite Reynolds numbers). The task of finding the leading singularities is precisely what the OPE was developed for.

However, we should warn the reader that what follows is essentially a self - consistent conjecture. In the case of statistical mechanics, when deriving hydrodynamics from the BBGKY equations it is necessary to assume the decrease of the correlations [7 ], a selfconsistent assumption that is difficult to prove from first principles. In our case this property is replaced by the OPE.

To understand how they work, let us reexamine the derivation of the previous equations. They were based on the fact that modulo the stirring force and the viscosity we have a sequence of conservation laws:

$$
\frac{\partial}{\partial t}\left(u^{n}\right)+\frac{n}{n+1} \frac{\partial}{\partial x}\left(u^{n+1}\right) \approx 0
$$

(The $\operatorname{sign} \approx$ here means that we don't write terms coming from the viscosity and the stirring force)

Equations (5) and (9) can be interpreted as relations for the generating functionals $\left\langle u^{n_{1}}\left(x_{1}\right) \ldots u^{n_{k}}\left(x_{k}\right)\right\rangle$. They involve both the stirring force and the viscosity. The former was already accounted for, while the latter presents a problem. The main rule of the game is that in any equation ,involving space points separated by the distance larger than $a$ viscosity can be set to zero. Thus it is perfectly legitimate to use the inviscid limit for $\mathrm{n}=1$, because in this case we exploit the steady state condition:

$$
\begin{array}{r}
\frac{d}{d t}\left\langle u\left(x_{1}\right) \ldots u\left(x_{N}\right)\right\rangle=0 \\
\left|x_{i}-x_{j}\right| \gg a
\end{array}
$$

However, starting from $n=2$ we have a problem, since in this case we have to take time derivatives of the product of $u-\mathrm{s}$ at the same point. To circumvent this problem in the case of $n=2$ let us replace:

$$
\begin{array}{r}
u^{2}(x) \Longrightarrow u\left(x+\frac{y}{2}\right) u\left(x-\frac{y}{2}\right) \\
\left|x_{i}-x_{j}\right| \gg y \gg a
\end{array}
$$


and let $y \rightarrow 0$ after the viscosity is taken to zero. In this case the use of the inviscid equations is justified, but we will get an anomaly in the conservation law, due to the point splitting. We have after simple algebra:

$$
\begin{gathered}
-\frac{d}{d t}\left(u\left(x+\frac{y}{2}\right) u\left(x-\frac{y}{2}\right)\right) \approx \frac{1}{2} \frac{\partial}{\partial x_{1}}\left(u^{2}\left(x_{1}\right) u\left(x_{2}\right)\right)+(1 \leftrightarrow 2) \approx \frac{2}{3} \frac{\partial}{\partial x} u^{3}(x)+a_{0}(x), \\
x_{1,2}=x \pm \frac{y}{2}
\end{gathered}
$$

Here we have introduced the first dissipative anomaly operator:

$$
a_{0}(x)=\lim _{y \rightarrow 0} \frac{1}{6} \frac{\partial}{\partial y}\left(u\left(x+\frac{1}{2} y\right)-u\left(x-\frac{1}{2} y\right)\right)^{3}
$$

In deriving this formula we set $y$ to zero inside all terms containing $x$-derivative. This is possible because all the correlation functions have finite $x$-dependent limit at zero $y$. We also used the identity:

$$
\frac{\partial}{\partial y}\left(u^{3}\left(x+\frac{y}{2}\right)-u^{3}\left(x-\frac{y}{2}\right)\right)=\frac{1}{2} \frac{\partial}{\partial x}\left(u^{3}\left(x+\frac{y}{2}\right)+u^{3}\left(x-\frac{y}{2}\right)\right) \rightarrow \frac{\partial}{\partial x}\left(u^{3}(x)\right)
$$

The anomaly would be zero if $u(x)$ were differentiable, since then the RHS of (17) is $\sim y^{2}$. However, the steady state condition dictates the opposite. Indeed, one of the consequences of eq. (5) is that in the steady state:

$$
\frac{d}{d t}\left\langle u^{2}\right\rangle=\kappa(0)-\left\langle a_{0}\right\rangle=0
$$

and hence we have the famous Kolmogorov relation:

$$
\left\langle\left(u\left(x_{1}\right)-u\left(x_{2}\right)\right)^{3}\right\rangle \propto \kappa(0)\left(x_{1}-x_{2}\right)
$$

The value of the anomaly defines the limiting contribution of the viscous term in the steady state:

$$
\lim _{\nu \rightarrow 0} \nu u(x) u^{\prime \prime}(x)=-a_{0}(x)
$$

An interesting feature of this relation is that it defines the expectation value of the $a_{0}$ - anomaly self-consistently from the steady state equation. This feature is preserved for the higher anomalies of the $u^{n}$ - densities. They are necessarily non-zero, because after point splitting procedure we get terms $\frac{\partial}{\partial y}\left(u\left(x+\frac{y}{2}\right)-u\left(x-\frac{y}{2}\right)\right)^{k}$ and the steady state equation will determine their value.

With a certain amount of vulgarization one can say that the reason for the $u^{n}$ anomalies is that shock waves absorb not only energy, but these higher densities as well.

Before computing the general anomaly let us discuss carefully all the limiting procedures involved. As we see correlation functions depend on the parameters $\kappa(0)=\varepsilon$, $L$, (which defines the correlation length of the forces)and the viscosity $\nu$. We made an assumption that as we let the viscosity go to zero correlation functions have a finite limit 
and hence depend only on $\varepsilon$ and $L$. This limit is what is meant by the inertial range. The standard Kolmogorov assumption (which we don't make) is that the Galilean - invariant correlation functions, such as $\left\langle\left(u\left(x_{1}\right)-u\left(x_{2}\right)\right)^{n}\right\rangle$ have a finite limit as $L \rightarrow \infty$. As we will see in our case this statement doesn't hold. Instead we have to make a different assumption, consistent with our equations. We will call it the G- (Galilean) assumption.

To formulate it let us notice that Galilean invariance in our system is spontaneously broken. This is evident from the fact that pumping forces create a certain average velocity $v_{r m s}=\sqrt{ }\left\langle u^{2}\right\rangle$. At the same time unbroken G-symmetry would require that the probability distribution be invariant under $u \Rightarrow u+$ const.

It is easy to estimate the value of $v_{r m s}$. In the limit of zero viscosity the only possible formula is :

$$
v_{r m s} \sim \kappa^{\frac{1}{3}}(0) L^{\frac{1}{3}} \sim \varepsilon^{\frac{1}{3}} L^{\frac{1}{3}}
$$

In the $\lambda$ representation this breakdown means that:

$$
\sum \lambda_{j} \sim \frac{1}{v_{r m s}}
$$

This absence of G-symmetry makes the anomaly computation difficult. Fortunately it is consistent with eq.(3) to assume that if we formally tend $L \rightarrow \infty$ and keep $\lambda_{j}$ finite, then G-symmetry is restored. We conjecture that in this limit:

$$
Z\left(\lambda_{1} x_{1} ; \ldots \lambda_{N} x_{N}\right) \propto \delta\left(\sum \lambda_{k}\right)
$$

This is the G-assumption and a short check shows that it is consistent with eq.(3). To state this assumption in a slightly more physical way, we can say that the probability distributions of velocities, $W\left(u_{1} x_{1} \ldots u_{N} x_{N}\right)$, which are Fourier transforms of the $Z$ functions, have the following structure:

$$
W\left(u_{1} x_{1} \ldots u_{N} x_{N}\right)=w\left(u_{i}-u_{j} ; x_{k}\right) W_{1}\left(\frac{\sum u_{k}}{v_{r m s}}\right)
$$

provided that :

$$
\left|u_{i}-u_{j}\right| \ll \sum u_{k}
$$

This last condition is very important. It is easy to see that without it the separation of the center of mass velocity which occured in (23) would contradict eq.(5).

The G-symmetry greatly simplifies computations of the anomaly. However one more self-consistent assumption is needed. This is an assumption of the existenceof an operator product expansion or the fusion rules. To formulate it we introduce the following notations:

$$
\begin{array}{r}
Z(\ldots)=\left\langle e_{\lambda_{1}}\left(x_{1}\right) \ldots e_{\lambda_{N}}\left(x_{N}\right)\right\rangle \\
e_{\lambda}(x)=\exp \lambda u(x)
\end{array}
$$


The fusion rules is the statement concerning the behavior of correlation functions when some subset of points are put close together. We conjecture that in our case the rules have the form:

$$
e_{\lambda_{1}}\left(x+\frac{y}{2}\right) e_{\lambda_{2}}\left(x-\frac{y}{2}\right)=A\left(\lambda_{1}, \lambda_{2}, y\right) e_{\lambda_{1}+\lambda_{2}}(x)+B\left(\lambda_{1}, \lambda_{2}, y\right) \frac{\partial}{\partial x} e_{\lambda_{1}+\lambda_{2}}+O\left(y^{2}\right)
$$

We will call this statement the F-conjecture. Here $A$ and $B$ are some functions to be determined and the meaning of (26) is that they control the fusion of the functions $Z_{N}$ into functions $Z_{N-1}$ as we fuse a couple of points together. To find the result one has to substitute (26) into (25). Of course one must check that this conjecture is consistent with the eq. (5), which is also is supposed to determine functions $A$ and $B$. To make this equation effective we have to evaluate the following anomaly operator:

$$
a_{\lambda}(x)=\lim _{\nu \rightarrow 0} \nu\left(\lambda u^{\prime \prime}(x) \exp \lambda u(x)\right)
$$

which appears on the right hand side. As we explained above, $a_{\lambda}(x)$ is generally non-zero, because smallness of $\nu$ is compensated by the blow-up of $u^{\prime \prime}(x+y) \exp \lambda u(x)$ as $y \rightarrow 0$. In fact we can write:

$$
a_{\lambda}(x)=\lambda \lim _{\nu, y, \zeta \rightarrow 0} \frac{\partial^{3}}{\partial \zeta \partial y^{2}} e_{\zeta}(x+y) e_{\lambda}(x)
$$

and exploit the F-conjecture to evaluate the RHS of (28). Thus, $a_{\lambda}(x)$ should be expressed in terms of derivatives of the functions $A$ and $B$. If the result is finite it must have the form:

$$
a_{\lambda}(x)=\alpha(\lambda) e_{\lambda}(x)+\tilde{\beta}(\lambda) \frac{\partial}{\partial x} e_{\lambda}(x)
$$

which is the only possible G-invariant expression,involving the ultraviolet finite operators $e(x)$ and $u^{\prime}(x)$. In order to have a finite limit in (28) one has to set the cut- off values of $y$ and $\zeta$ to be dependent on $\nu$ and $\lambda$. This is not surprising, since $\frac{1}{\lambda}$ defines the local Reynolds number and the ultraviolet cut-off must depend on it. It is also worth stressing again that this form of the anomaly is correct only in the Galilean- invariant limit. For generic $\lambda_{j}$ we would obtain a superposition of exponents with different $\lambda$, a rather difficult situation to treat.

The master equation (5) now takes the form (for the steady state):

$$
\begin{gathered}
H Z \equiv \sum\left(\frac{\partial}{\partial \lambda_{j}}-\beta\left(\lambda_{j}\right)\right) \frac{\partial}{\partial x_{j}} Z-\sum \kappa\left(x_{i}-x_{j}\right) \lambda_{i} \lambda_{j} Z=\sum \alpha\left(\lambda_{j}\right) Z \\
\beta(\lambda)=\tilde{\beta}(\lambda)+\frac{1}{\lambda}
\end{gathered}
$$


It is now a simple matter to check our F-conjecture. If we introduce the variables:

$$
\begin{gathered}
x_{1,2}=x \pm \frac{y}{2} \\
\lambda=\lambda_{1}+\lambda_{2} \\
\mu=\lambda_{1}-\lambda_{2}
\end{gathered}
$$

and keep $y$ much smaller than all other distances, we find the following structure of the operator $H$ :

$$
\begin{gathered}
H_{N}=H_{N-1}+F \\
F=2 \frac{\partial^{2}}{\partial y \partial \mu}-\left(\beta\left(\lambda_{1}\right)-\beta\left(\lambda_{2}\right)\right) \frac{\partial}{\partial y}+\left(\beta(\lambda)-\frac{1}{2}\left(\beta\left(\lambda_{1}\right)+\beta\left(\lambda_{2}\right)\right) \frac{\partial}{\partial x}\right.
\end{gathered}
$$

Here the operator $H_{N-1}$ is obtained from $H_{N}$ by replacing the points $x_{1,2}$ with the point $x$ and $\lambda_{1,2}$ with $\lambda=\lambda_{1}+\lambda_{2}$. From this we derive equations for the functions $A$ and $B$ that appear in (26):

$$
\begin{gathered}
\left(\nabla_{1}-\nabla_{2}\right) \frac{\partial B}{\partial y}=\frac{1}{2}\left(\beta\left(\lambda_{1}\right)+\beta\left(\lambda_{2}\right)\right)-\beta\left(\lambda_{1}+\lambda_{2}\right) \\
\left(\nabla_{1}-\nabla_{2}\right) \frac{\partial A}{\partial y}=\alpha\left(\lambda_{1}\right)+\alpha\left(\lambda_{2}\right)-\alpha\left(\lambda_{1}+\lambda_{2}\right),
\end{gathered}
$$

Here:

$$
\nabla=\frac{\partial}{\partial \lambda}-\beta(\lambda)
$$

These equations have solutions for any functions $\alpha$ and $\beta$. The next step is to substitute these solutions back in eqs. (28) and (29) and try to find constraints on $\alpha$ and $\beta$. Surprisingly the arising constraints are very weak due to the possibility to adjust the cut-off's, and thus the functions $\alpha$ and $\beta$ in (29) remain almost arbitrary. In principle they must be determined from the conditions that all probability distributions have admissible behaviour at $\infty$, much in the same way in which eigenvalues are usually determined. Since we don't have any general methods for treating this problem we will simplify the matter even more by introducing a scaling conjecture ( S-conjecture) which again turns out to be self- consistent. To formulate it let us notice that if $x \ll L$ we can expand $\kappa(x)=\kappa(0)\left(1-\frac{x^{2}}{L^{2}}\right)$ and in (30) the constant part, $\kappa(0)$ drops out due to the G-invariance:

$$
\begin{array}{r}
\sum \kappa\left(x_{i}-x_{j}\right) \lambda_{i} \lambda_{j}=\kappa(0)\left(\sum \lambda_{i}\right)^{2}+O\left(x^{2}\right) \\
\sum \lambda_{i}=0
\end{array}
$$

Therefore it is natural to look for a scaling solution with $\lambda \sim \frac{1}{x}$. The scaling condition determines the possible form of the functions $\alpha$ and $\beta$. In order to conform to scaling, they must be as following: 


$$
\begin{aligned}
& \beta(\lambda)=\frac{b}{\lambda} \\
& \alpha(\lambda)=a
\end{aligned}
$$

We will see now that scaling is self-consistent, although one can also try more general solutions, say with logarithmic terms.

Let us see how the unknown numbers $a$ and $b$ are determined from the eigenvalue problem. For example consider a 2-point function. The master equation in this case takes the form:

$$
\left(\frac{\partial}{\partial \mu}-\frac{2 b}{\mu}\right) \frac{\partial}{\partial y} Z+\mu^{2} y^{2} Z=a Z
$$

For reasons to be clarified later we are interested in the case $a=0$. Our S-conjecture amounts in the anzats:

$$
Z(\mu, y)=\Phi(\mu y)
$$

Here we temporarily use the units in which $\kappa(0)=1$ and $L=1$ The function $\Phi(x)$ satisfies an ordinary differential equation:

$$
x \Phi^{\prime \prime}(x)+(1-2 b) \Phi^{\prime}(x)+x^{2} \Phi(x)=0
$$

The general solution of this equation has the form:

$$
\Phi(x)=x^{b} F_{\frac{2 b}{3}}\left(\frac{2}{3} x^{\frac{3}{2}}\right)
$$

where $F_{\lambda}$ is one of the Bessel functions. The right function and the value of $b$ are determined from the condition that the probability distribution:

$$
w(u, y)=\int_{c-i \infty}^{c+i \infty} \frac{d \mu}{2 \pi i} Z(\mu, y) e^{-\mu u}
$$

must be positive and vanish as $u \rightarrow \pm \infty$. From the convergence of (38) it follows that we must chose

$$
\Phi(x) \propto x^{b} K_{\frac{2 b}{3}}\left(-\frac{2}{3} x^{\frac{3}{2}}\right)
$$

Positivity of $Z$ for $x>0$ and its finitness at $x=0$ forces us to take $b=\frac{3}{4}$ for which case:

$$
\Phi(x)=\exp \frac{2}{3} x^{\frac{3}{2}}
$$

As a result we obtain the following result for the probability to have a velocity difference $u$ at the distance $y$ :

$$
w(u, y)=\int_{c-i \infty}^{c+i \infty} \frac{d \mu}{2 \pi i} \exp \left[\frac{2}{3}(\mu y)^{\frac{3}{2}}-\mu u\right]
$$


The positivity of $w$ is guaranteed by the fact that $Z$ satisfies certain convexity conditions. Indeed, from the relation:

$$
Z(\mu)=\int_{-\infty}^{\infty} w(u) e^{\mu u}>0
$$

we derive, by using Cauchy inequality:

$$
Z\left(\mu_{1}\right) Z\left(\mu_{2}\right)>Z^{2}\left(\frac{\mu_{1}+\mu_{2}}{2}\right)
$$

which is clearly satisfied. It is mathematically curious that the $u$ representation the eq.(34) is easily reduced to the Schroedinger equation with the potential $V(u)=\frac{u^{4}}{4}-2 b u$. This is achieved by the change:

$$
Z(\mu, y)=(\mu)^{2 b} F(\mu, y)
$$

, which removes the $\frac{1}{\mu}$ term in the eq. (34) and by the Fourier transform to the function $\tilde{F}(u, y)=y^{2 b-1} \phi\left(\frac{u}{y}\right)$. The power of $y$ here is needed for consistency with (35). The value of $b$ corresponds to the zero energy eigenvalue in this potential and $\phi$ is proportional to the ground state wave function 1 .

The probability distribution defined by (41)has the following asymptotic behaviour:

$$
w(u, y)= \begin{cases}e^{-\frac{1}{3}\left(\frac{u}{y}\right)^{3}} & \text { if } \frac{u}{y} \rightarrow+\infty \\ y^{\frac{3}{2}} u^{-\frac{5}{2}} & \text { if } \frac{u}{y} \rightarrow-\infty\end{cases}
$$

This qualitatively fits the observations [5 ]. It must be stressed however that some caution is needed when comparing the G-invariant part of the probability distributions with the experiment. As we have already said, the factorization (24) breaks down at large velocities. That means in particular that in general $w(u, y)$ has the following structure:

$$
w(u, y)=\chi\left(\frac{u L}{v_{r m s} y} ; \frac{u}{v_{r m s}} ; \frac{y}{L}\right)
$$

The scaling limit, discussed above is reached only when two conditions are satisfied:

$$
y \ll L ; u \ll v_{r m s}
$$

When computing the moments of the probability distributions, which represent correlation functions, it may happen that even when the first condition is enforced, the second one will be violated. In this case the result is not universal, since the behaviour of probabilities at $u \sim v_{r m s}$ depends on the correlations of the stirring forces at $x \sim L$.

We come to the conclusion that the breakdown of Galilean invariance leads to a rather peculiar structure of the correlation functions. They contain in general both universal and non-universal parts. The former comes from the distribution (43) and its generalization for

\footnotetext{
${ }^{1}$ A. Migdal informed me that he was able to find this wave function directly from the Schroedinger equation
} 
an arbitrary number of points. The latter results from the region of the large velocities. These nonuniversal correlations are just the "condensates" introduced in ref.[1]. The formula (43) shows that due to the "power tail" all the expectation values of $\left\langle u^{n}\right\rangle$ starting with $n=\frac{3}{2}$ are formally divergent. That simply means that they are dominated by the non-universal region and thus change if we change $\kappa(x)$ for $x \sim L$. At the same time, the moments with $n<\frac{3}{2}$ are universal. The "power tail" in the formula (43) must be related to the probability of having a kink, introduced in ref.[5]. However the precise connection is not completely clear, since the non-universal part may be relevant in the comparison.

We come to the conclusion that at least in the present setting the violation of the naive scaling for higher moments - the phenomenon usualy called "intermittency"- is due to the breakdown of Galilean symmetry and non-universality of the large velocity fluctuations. In the past "intermittency" essentially meant that the theory sometimes works and sometimes doesn't. Here we have it under control. This observation explains an apparent discrepancy between the scaling in the eq. (41) and Kolmogorov's relation (19). These two come from the different regions of the phase space. This is evident from the fact that the value of $\kappa(0)$ which enters into Kolmogorov's relation (19) simply drops off in the G-invariant limit, as seen from (33). For the different type of the stirring forces considered in [5] the two regions seem to overlap. That forms the basis for the beautiful physical picture advocated in [5]. It is also consistent with the approximate solution of the Burgers problem found by the replica method in [6].

In the above solution we took $a=0$. Our understanding of other possible solutions is still incomplete, although it seems that for the considered type of stirring forces an attempt to take $a \neq 0$ leads to some unphysical results, like having $\left\langle u\left(x_{1}\right)-u\left(x_{2}\right)\right\rangle \neq 0$. However for a different type of stirring forces which lead to different scaling laws we almost certainly have to include the $a$ term. This question is currently under investigation [ 8].

Finally, let us present the generalization of the master equation for arbitrary dimensionality. In the inviscid limit it isn't difficult. Consider the following quantity:

$$
\Theta_{\vec{\lambda}}=\rho(x, t) e^{\vec{\lambda} \vec{v}(x, t)}
$$

where $\rho$ and $\vec{v}$ are the density and the velocity, satisfying the Euler equations:

$$
\begin{gathered}
\dot{\rho}+\partial_{\alpha}\left(\rho v_{\alpha}\right)=0 \\
\rho\left(\dot{v}_{\alpha}+\left(v_{\beta} \partial_{\beta}\right) v_{\alpha}\right)=f_{\alpha}
\end{gathered}
$$

It is straightforward to verify by the same methods which led to eqs. (5) and (9) that the correlation function of the $\Theta_{\vec{\lambda}}$ 's :

$$
F=F\left(\vec{\lambda}_{1}, \vec{x}_{1} ; \ldots \vec{\lambda}_{N} \vec{x}_{N}\right)
$$

satisfies the following equation:

$$
\dot{F}+\sum \frac{\partial^{2} F}{\partial \overrightarrow{\lambda_{j}} \partial \overrightarrow{x_{j}}}=\sum \kappa_{\alpha \beta}\left(\vec{x}_{i}-\vec{x}_{j}\right) \lambda_{i \alpha} \lambda_{j \beta} F
$$


which generalizes eq.(8) for an arbitrary dimension. As in one dimension the origin of this equation lies in the special conservation laws analogous to (13). In general we have the following set of conserved tensors:

$$
T_{\alpha_{1} \ldots \alpha_{n}}=\rho v_{\alpha_{1}} \ldots v_{\alpha_{n}}
$$

which satisfy a continuity equation.

The next step should be an analyses of anomalies along the same lines as above. This task is not completed yet. Another immediate problem is to include the pressure as a small perturbation. This is possible to do by using the relation:

$$
\rho(\vec{x})=\left.\Theta_{\vec{\lambda}}(\vec{x})\right|_{\vec{\lambda}=0}
$$

This relation allows us to express the perturbations of pressure and density in terms of the function $F$. However this analyses is also a problem for the future.

I am deeply grateful to V. Borue for useful discussions, A. Migdal and V.Yakhot for sharing with me their insights, results and enthusiasm concerning turbulence, and to D. Gross for his important critical remarks on physics and style of this article.

This work was partially supported by the National Science Foundation under contract PHYS-90-21984. 


\section{REFERENCES}

[1] A.Polyakov Nucl. Phys. B396 (1993) 367

[2] Ya. Zeldovich Astron. and Astroph. 5 (1972) 84

[3]M.Kardar et al. Phys.Rev. Lett.56 (1986) 889

[4] Ya. Sinai Journal of Stat. Phys.84(1991) 1

[5] A. Chekhlov, V.Yakhot Phys. Rev E 51(1995) R2739 and to be published

[6] J. Bouchaud et al. LPTENS preprint 95/12

[7] E. Lifschitz L. Pitaevsky 'Physical Kinetics' Pergamon Press (1981)

[8] A. Migdal A. Polyakov V. Yakhot in preparation 